\title{
Keypoint detectors and texture analysis based comprehensive comparison in different color spaces for automatic detection of the optic disc in retinal fundus images
}

\author{
Kemal Akyol ${ }^{1}$ D $\cdot$ Baha Şen $^{2}$ \\ Received: 2 May 2020 / Accepted: 4 August 2021 \\ Published online: 14 August 2021 \\ (c) The Author(s) 2021 OPEN
}

\begin{abstract}
Detection of the optic disc which has similar brightness with the hard and soft exudate lesions seen in the early stage of diabetic retinopathy is very difficult due to different light conditions and contrast values. Automatic detection of these lesions by expert systems in the medical field is very important. In this context, we propose a new approach based on the analysis of color spaces, keypoint detectors, and texture for retinal fundus images. If the keypoint information is contained within the actual optic disc region, this is an important consideration for the automated detection of the optic disc. This study can be divided into five sections, respectively, image preprocessing, image processing, keypoint detection, texture analysis, and performance evaluation. The analyses of patch images compatible with the keypoints obtained from the Red-Green-Blue (RGB) image and its color channels were carried out. The performance of the study was validated on the Digital Retinal Images for Vessel Extraction public dataset. According to the results, Local Binary Pattern texture analysis performed in region of interest around keypoints detected by different keypoint detectors presented good performance in RGB and green channel images.
\end{abstract}

\section{Article Highlights}

- Optic disc detection is an important prerequisite for the diagnosis of retinal diseases.
- Accurate detection of the optic disc is very difficult because of eye diseases, light, and noise.

- Keypoints play an important role in order to detect optic disc in the retinal fundus image.

Keywords Optic disc · Keypoint detection · Texture analysis · Error distance · Jaccard index

\section{Introduction}

The optic disc (OD) is the main region in retinal images. The coordinates of this region are need detected in order to determine other retinal structures such as macula and blood vessels [1]. OD is similar to hard and soft exudates with respect to color and brightness features [2]. Therefore, the detection of this region in the retinal images with the anomaly is particularly difficult. OD is the exit point of all vessels that spread to the retinal fundus image and this region is different from other bright regions. The characteristics of an optic disc region are generally as follows [3]:

(a) Approximately circular

\footnotetext{
$\triangle$ Kemal Akyol, kakyol@kastamonu.edu.tr|' Department of Computer Engineering, Faculty of Engineering, Kastamonu University, Kastamonu, Turkey. ${ }^{2}$ Department of Computer Engineering, Faculty of Engineering and Natural Sciences, Ankara Yıldırım Beyazıt University, Ankara, Turkey.
}

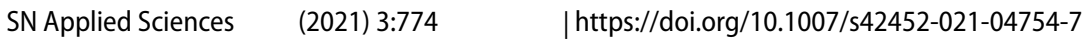


(b) Color tints between yellow and orange.

(c) Brightness value usually higher than the rest of the retinal image

(d) A region less than $1 / 7$ of the entire retinal image

Accurate detection of the $\mathrm{OD}$ is very difficult because of eye diseases, light, and noise [4]. Retinal structure deteriorations cause difficulty in the automatic detection of the optic disc. For example, it is seen that two retinal fundus images have different brightness values in Fig. 1. Therefore, the fail predictions or detections are inevitable.

Well-done segmentation of this region is a very important and necessary step in order to analyze and diagnose many eye-related diseases in computer-aided diagnosis systems $[2,5]$. The detection of glaucoma eye disease is related to tracing the changes in the optic cup. This region must be detected in the retinal image [6] due to it enables ophthalmologists to mitigate the effects of these diseases [7].

The motivation of this study is to propose a model that helps detect the optic disc region This paper presents new research for the detection of the optic disc in the retinal fundus image. With this aim, this study consists of the steps below performed on the Red-Green-Blue (RGB) image and its color spaces:

(a) Pre-processing the retinal fundus image

(b) Detecting keypoints

(c) Comparing and evaluating the patch images by utilizing Local Binary Pattern (LBP).

It is noted that Akyol et al. [8] carried out the OD detection by using keypoint detection, texture analysis, and visual dictionary methods only on the RGB images in their study. The study of [8] promotes the use of keypoint for detecting of the optic disc. In light of the [8]'s study that encourages the use of the keypoint for better perception of the optic disc, a comprehensive comparison based on keypoint detection algorithms and texture analysis in
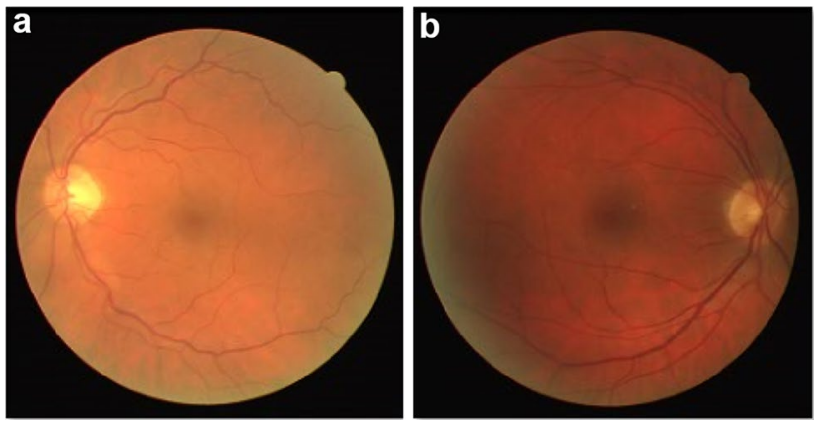

Fig. 1 Retinal fundus images; a a sample image having a maximum brightness, $\mathbf{b}$ a sample image having a minimum brightness different color spaces was carried out in the proposed study. In here, the Local Binary Pattern texture analyses of patch images compatible with the keypoints detected with Oriented FAST and rotated BRIEF (ORB) [9], ScaleInvariant Feature Transform (SIFT) [10], and Speeded up robust features (SURF) [11] keypoint detection algorithms on the RGB image and its color channels were carried out.

(a) Distinctly from the study of [8];

(b) In this study, wavelet denoising was applied in the image preprocessing process.

(c) Experiments were carried out with different color spaces.

(d) Experiments were conducted on the different keypoint detection algorithms.

(e) While the Local Binary Pattern similarity score was used, and the most similar patch image was detected in this study, the Random Forest classifier was used in [8]'s study.

Hence, this study differs from the [8] within the frame of the workflow steps.

The rest of this paper is structured as follows: Sect. 2 presents the literature review. Section 3 describes the materials and methods. Sections 4 and 5 present the experiments and results, respectively. Section 7 discusses the proposed approach and other studies. Finally, a conclusion is drawn in Sect. 7.

\section{Literature review}

As can be seen in literature, there is a considerable number of studies for the detection of the OD region. Muhammed presented different optic disc localization algorithms based on the assumption of optic disc area which has rich information, so its entropy value is more significant in this area. The entropy of different patch images obtained by the sliding window technique was analyzed using recursive steps in their study [6]. Allam et al. focused on this subject with three categories: (a) using the size, color, and brightness characteristics (b) forecasting the convergence point of the vessels (c) template matching [7]. Lalonde detected OD by template matching based on pyramidal decomposition and Hausdorff in low-resolution fundus images [12]. Park et al. (2006) used simple image processing algorithms including threshold, detection of object roundness, and circle detection by Hough transformation in order to automatically detect outline the optic disc [13]. Mahfouz and Fahmy proposed a study on determining the $O D$ region by using image features on the publicly available datasets such as STARE and DRIVE [14]. Zhu et al. automatically located the optic nerve head by using 
edge detection and circle detection. They utilized Sobel operators and Hough transform for edge detection and circle detection, respectively [15]. Whardana and Suciati [16] used the combination of two techniques which consist of morphological operators and k-means clustering. Abdullah et al. presented a study including morphological operations, circular Hough transform, and grow-cut algorithm. First, they focused on the optic disc region by applying morphological operators. Then, they calculated the optic disc center using the circular Hough transform approximately. Lastly, they used the grow-cut algorithm in order to precisely detect the optic disc boundary [17]. Hamednejad and Pourghassem applied the masking procedure on the image in order to determine candidate ROls. Then, they used combinational filtering and converted the region of interest image to the $L^{*} a^{*} b^{*}$ color space. After that, they used density-based spatial clustering applications for OD segmentation and recognition [18]. Ichim and Popescu [19] extracted some features by utilizing adaptive local texture analysis and classified them. Roychowdhury et al. applied morphological operations on the green channel image in order to extract bright regions. After, they classified these regions as OD and non-OD using six region-based features and a Gaussian mixture classifier [20]. Park et al. (2017) presented an automated technique in order to detect optic disc region by utilizing a set of image and geometric processing techniques. Experiments in their study were performed on several public fundus images. Their proposed algorithm was successful on the fundus images of healthy patients [21]. Sharma et al. presented a study in order to detect the optic nerve head by using many image features. This study was evaluated on the six public datasets consisting of normal and diseased retinal images [22]. Sudhan et al. presented a study on the heuristic algorithm assisted multi-level thresholding and level set-based segmentation of OD on the RIM-ONE retinal image dataset [23]. Zahoor and Fraz localized OD fastly and accurately by using morphological operations and circular Hough transform. Also, they obtained OD boundary information using a novel polar transform-based adaptive thresholding [24]. In [25], the authors handled out this subject by three categories. a) shape and template matching b) using the active contours and different energies in the image $\mathrm{c}$ ) extracting the features and using the machine and deep learning algorithms. They presented a new deep multiscale sequential convolutional neural network-based study for the segmentation of OD and optic cup on the Messidor and Kaggle datasets. Fan et al. trained the supervised model by using the OD patch image. And this model presents an edge map for the OD. Threshold and then the circular Hough transform was applied to this edge map image in order to detect approximate OD boundary [26]. Hou et al. detected OD automatically by utilizing template matching. They used best-buddies similarity measure in order to calculate the similarity between patch images obtained from retinal fundus image and hand-marked OD region [27]. Nergiz et al. proposed an approach based on three steps: (a) To roughly localize OD, finding the approximate convergent point of the vessels. (b) Extracting three features and designing a fuzzy logic [28]. Lastly, Díaz-Pernil et al. [29] detected the OD on the DRIVE and DIARETDB1 datasets by utilizing graphics processing unit technology. Their study consists of accelerated graphics port-color segmentation, Hamadani's binarization technique, and Hough circle cloud, respectively. Recently, deep learning-based models have been carried out for this subject. Mitra et al. classified the retinal images in publically available MESSIDOR and Kaggle datasets by utilizing the Convolution Neural Network in order to predict bounding boxes associated with their similar probabilities and confidence scores [2]. Calimeri et al. used GoogleNet pre-trained convolution neural network in order to detect the OD region [30]. Yu et al. used a modified U-Net architecture that combines pre-trained ResNet-34 and classical U-Net for optic disc and cup segmentation [31]. Finally, Wang et al. detected the optic disc by using a coarse-to-fine deep learning framework on the basis of a classical convolutional neural network [32].

\section{Material and methods}

\subsection{Material}

The performance of the study was tested on the Digital Retinal Images for Vessel Extraction (DRIVE) public dataset. The dataset is set of 40 retinal fundus images with size of [ $565 \times 584$ ] pixels obtained from a diabetic retinopathy monitoring program [33].

\subsection{Methods}

\subsubsection{Image processing}

The work steps performed on each image for more successful detections are as follows:

a. Wavelet denoising

b. Image channels extraction (obtained Red, Green and Blue channels).

c. Contrast-limited adaptive histogram equalization (CLAHE) [34] and Gamma correction [35] methods respectively to RGB image and its color channels.

d. Applying keypoint detection algorithm on the result images obtained from previous step. 
e. Texture analyze based on local binary pattern computation.

\subsubsection{Keypoint detection and texture analysis}

Keypoints represent the significant points on the image. Keypoint detection algorithms detect the keypoints which represent the information for the patch images. With the think that keypoints may occur on the optic disc region, this study was performed. LBP texture analysis firstly introduced by Ojala et al. [36] was performed in this study. The performance of the study was validated on the DRIVE public dataset.

\subsubsection{Evaluation}

In this study, the Error Distance (ED) and the Jaccard Index (JI) metrics were used in order to compare the detected and the manually determined optic disc region. ED is the distance calculation between the predicted OD center and the OD ground-truth center. Euclid formula as given in Eq. 1 is used widely for this calculation.
$E D=E D(G T x y, P x y)=\sqrt{(G T x-P x)^{2}+(G T y-P y)^{2}}$

where GT and P are the center coordinates of the actual optic disc region and predicted optic disc region, respectively. $x$ and $y$ represent the pixel coordinates.

The JI metric named as similarity index is used for evaluating the performance of the proposed method. $\mathrm{Jl}$ is a coefficient resulting in the ratio of similarity of two samples compared. A higher value of $\mathrm{JI}$ indicates that the estimated and real values look more alike to each other. When these two results are equal to each other, the Jl value is 1 , and when the two results haven't any common features, it is 0 . In other words, the $\mathrm{Jl}$ has a value between 0 and 1. The $\mathrm{Jl}$ is given in Eq. 2 . In this equation, $A \cap B$ indicates the region of intersection and $A \cup B$ indicates the region of union between two sets $A$ and $B$ [37]. Based on this equation, Pseudocode 1 was used in the experiments for the JI calculation.

Jaccard index $(A, B)=\frac{A \cap B}{A \cup B}$

Pseudocode 1. Jaccard index calculation

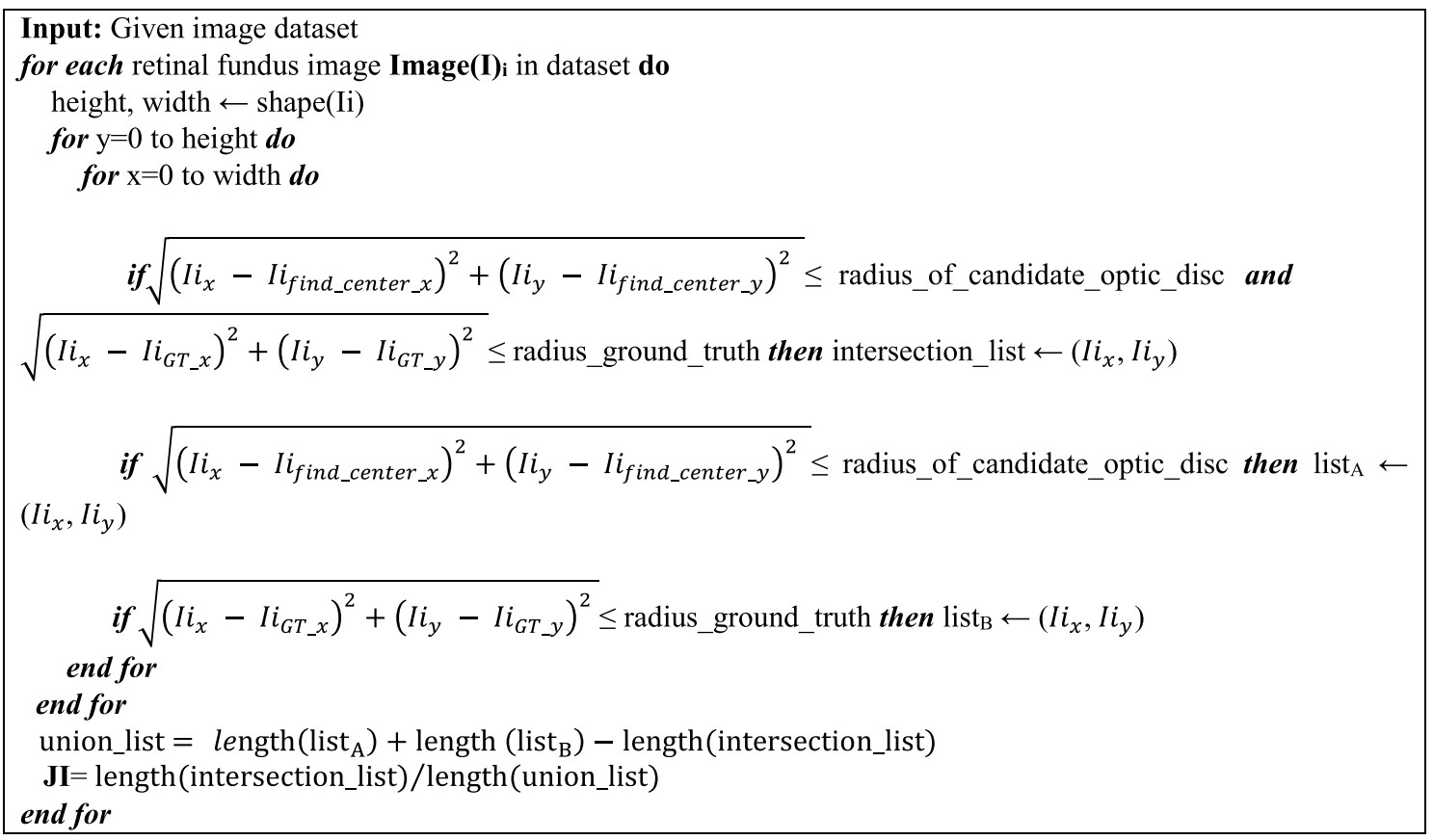

\section{Experiments}

In this study, a comprehensive analysis of keypoint detectors and texture analysis on the retinal fundus image and its color channels for automated detection of the optic disc were carried out. As can be seen in Fig. 2, the proposed approach consists of image pre-processing, image processing, keypoint detection, texture analysis, 


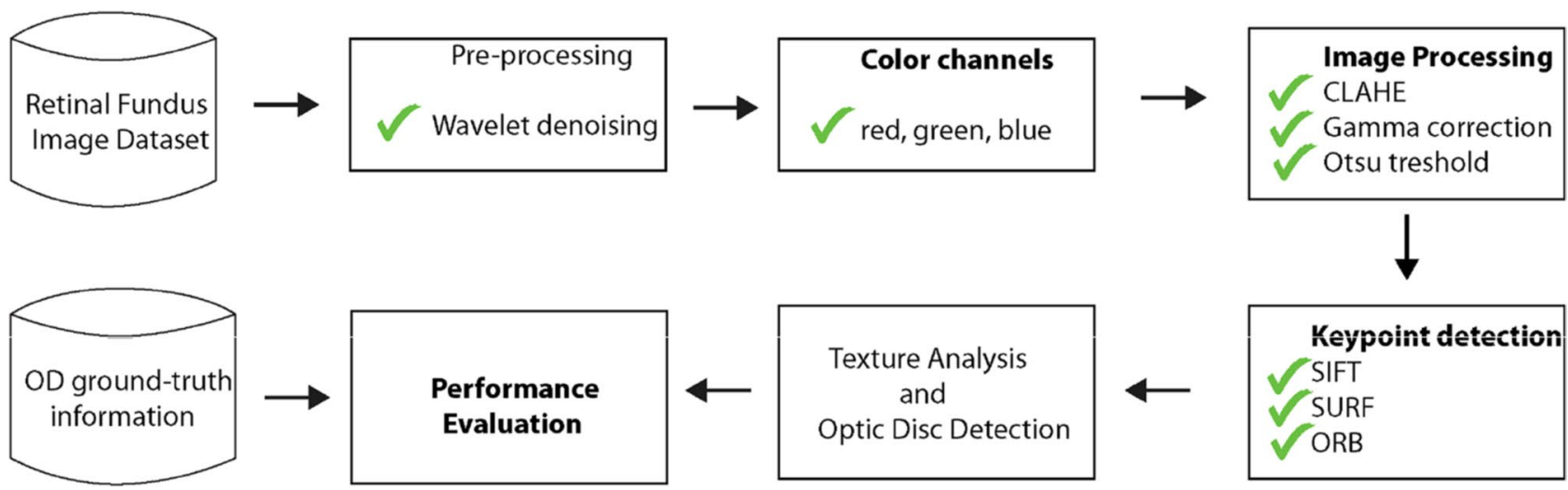

Fig. 2 The flowchart of the proposed approach

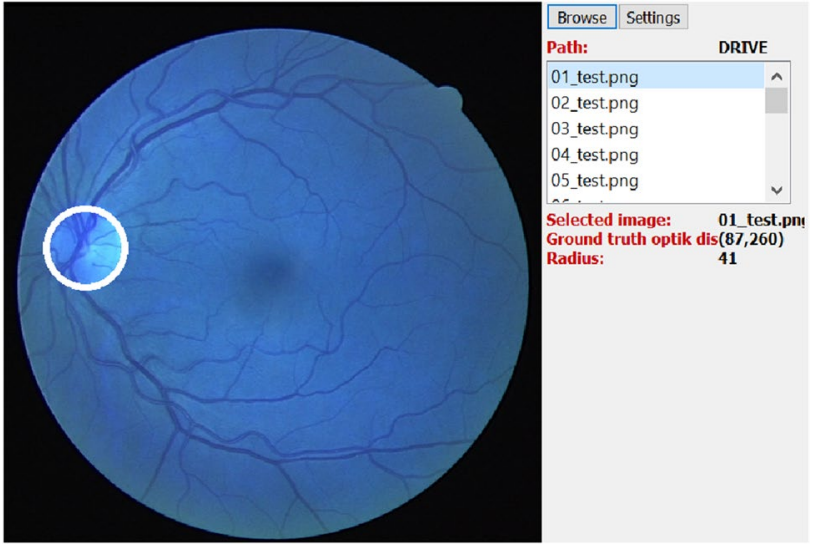

Fig. 3 The determination of ground-truth and radius information for an optic disc

and performance evaluation generally. These steps are explained in detail below.

To compare the detected and actual OD centers, first, the OD ground-truth center and the radius information in each retinal image were stored by the ophthalmologist Hasan Basri Çakmak with marking the circles manually using an application developed given in Fig. 3.

The processes involving all steps were given in Fig. 4, respectively.

(a) Image pre-processing techniques:

a.1 Wavelet denoising was applied to all original images in order to remove the noise (Fig. 4a). Then, the red, green, and blue channels of all images were extracted by color space transformation and they were stored in the system (Fig. 4b).

a.2 CLAHE method was applied to all images for improving the brightness and contrast (Fig. 4c).

a.3 Gamma correction was implemented on all images obtained by the CLAHE method (Fig. 4d). a.4 Otsu threshold algorithm was applied to all images obtained by Gamma correction for the binarization of the images. And so, the result images were obtained for performing keypoint detection.

(b) Detection of keypoints and analysis of candidate patch image: As seen in Fig. 4e and f, the SIFT, SURF, and ORB algorithms were applied to these result images; RGB image and its color channels. The region of interest (ROI) size around these keypoints was determined in accordance with its optic disc ground truth radius pixel value predefined by ophthalmologist considering the interface in Fig. 3 for each image. The answer to the question of "is the ROI patch image an optic disk region?" was queried for each $\mathrm{ROI}$ regarding keypoint by thorough LBP texture analysis.

As can be seen in Fig. $4 \mathrm{~g}$ and $\mathrm{h}$, the red circle shows the candidate $O D$ region, and the blue circle shows the actual OD region. So, all patch images compatible with all keypoints were examined. Then the information of the region which has the best $\mathrm{JI}$ score and the least ED value was stored in the system in the dictionary structure.

With the result of comparing twenty optic disc and nonoptic disc patch images with equally in the system, optic disc labeling for the patch image was performed and the ED and JI measures were calculated. The best candidate optic disc region was tried to find by applying this process to all patch images compatible with keypoints. Pseudocode 2 and Pseudocode 3 present the processes essential for candidate optic disc detection and performance evaluation, respectively. The developed application first determines the candidate optical disc regions according to the LBP information and decides that one of them having the best $\mathrm{Jl}$ similarity score and the smallest ED value is the optic disc. 


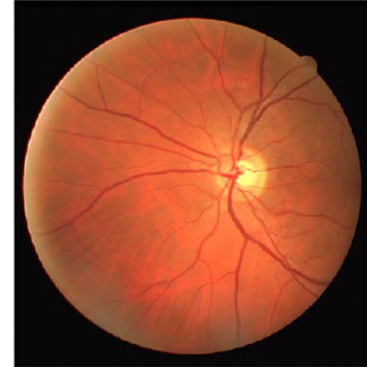

a Retinal fundus image obtained by Wavelet denoising

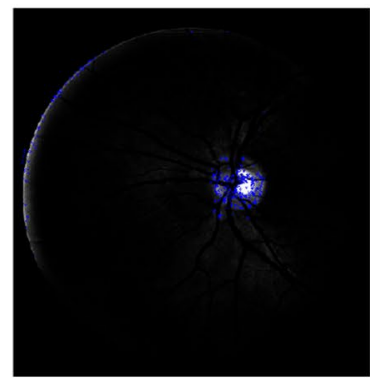

e Keypoints on the image obtained by SURF method

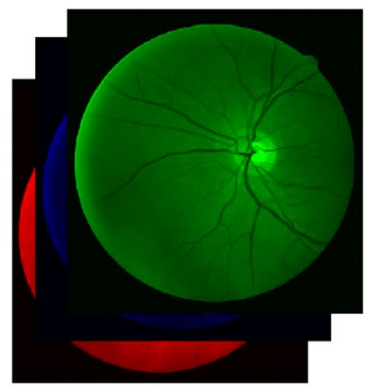

b $R, B$ and $G$ channels

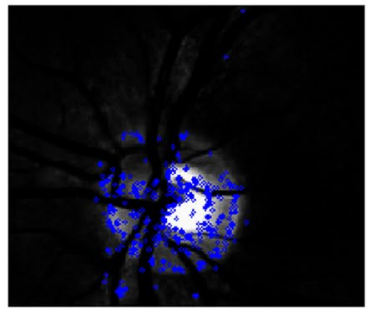

f Keypoints on the optic disc region (zoom of optic disc region)

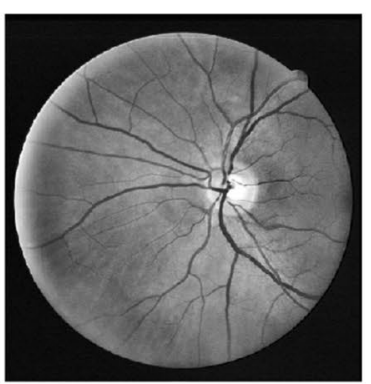

c An image obtained by CLAHE method

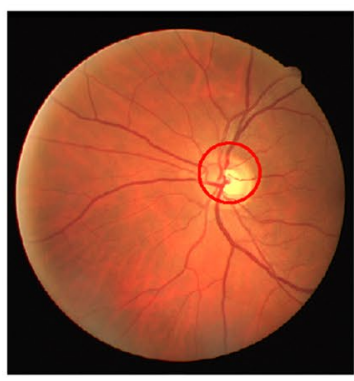

g Detected OD region on the image (red circle)

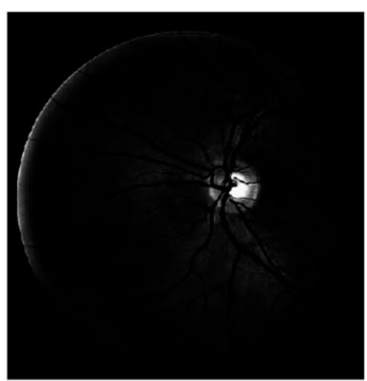

d An image resulted from Gamma correction and Otsu algorithm

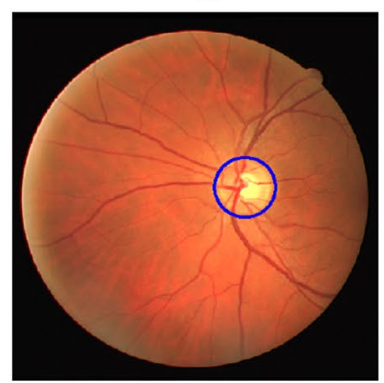

h Actual optic disc region (blue circle)

Fig. 4 Steps of the optic disc detection applied in this study

Pseudocode 2. Candidate optic disc detection

\begin{tabular}{|l}
\hline Require: Region of Interest (patch image) ROI; target image I. \\
foreach retinal fundus image Ii in dataset do \\
Preprocess for Ii \\
Color channels for Ii \\
foreach keypoint algorithm Kp in ['ORB', 'SIFT', 'SURF'] do \\
foreach RGB and color channels for each retinal fundus image do \\
keypoint_list : Detect the keypoints on the image \\
foreach keypoint in keypoint_list do \\
Detect region of interest (patch image) corresponding with keypoint \\
Compute similarity score (Compute LBP measure for each patch image) \\
Find best score \\
end for \\
end for \\
end for \\
end for
\end{tabular}


Pseudocode 3. Performance evaluation

Best result: The location of the region based on the best result
foreach retinal fundus image Ii in dataset do
foreach $\mathrm{R} G \mathrm{~B}$ and color spaces for each retinal fundus image do
foreach keypoint algorithm in ['ORB', 'SIFT', 'SURF'] do
Calculate the $E \boldsymbol{E}$ as the distance between candidate optic disc and ground
truth optic disc using Equation (1)
Calculate the $\boldsymbol{J I}$ similarity score
Evaluate the performance of the keypoint algorithm for each image
end for
end for
end for

One of the following three cases was assigned to each retinal image automatically in the proposed study.
(a) OD detected
(b) OD detected partly
(c) OD not detected

The OD position is accepted correctly detected if the estimated coordinates are inside the contour of the OD, i.e., within 60 pixels of its center, as manually identified for ground truth in [1,34]. In our study, differently from these studies in the literature, if the ED is equal to OD groundtruth radius or less than, the algorithm is considered successful for finding the optic disc. This process automatically terminates after all patch images were analyzed. Figure 5 demonstrates that some of the successful and unsuccessful OD detection results. Figure 5 a demonstrates that the success achieved is very high. Figure $5 \mathrm{~b}$ shows that a part of the optic disc detected. Figure $5 c$ says that the optic disc detection isn't accurate because the ED between two centers measure is bigger than the radius value. Pseudocode 4 presents the calculation of the average success ratio of the proposed system. As seen here, one of the three values, Successful Detected, Failed Detected, Non-Detected, was assigned to any retinal image analyzed. The Successful Detected (positive) and Failed Detected (negative) were calculated using the ED and JI metrics. The number of ND is included for the calculation of the average success ratio for optic disc detection.
Fig. 5 Some optic disc detection results
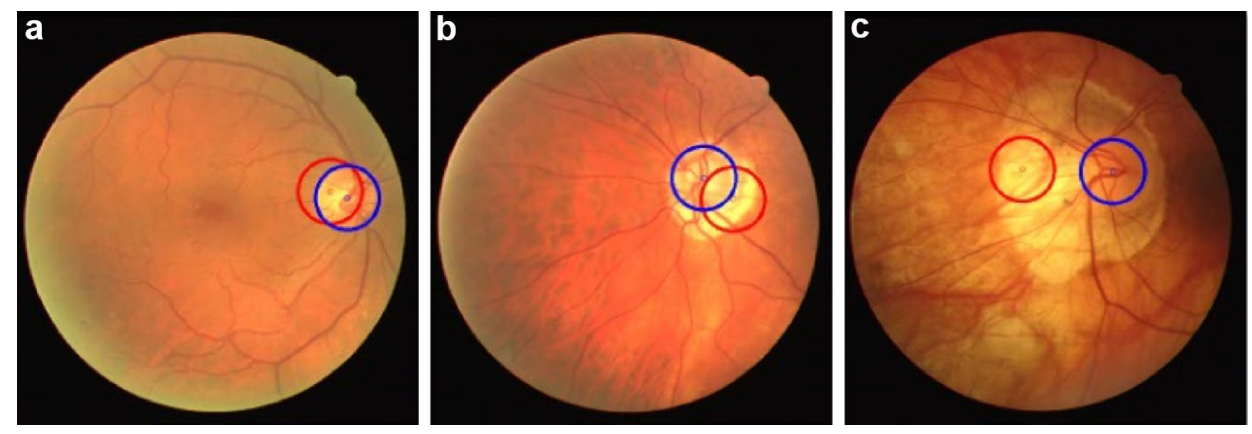
Pseudocode 4. Calculation of the success ratio for OD detection

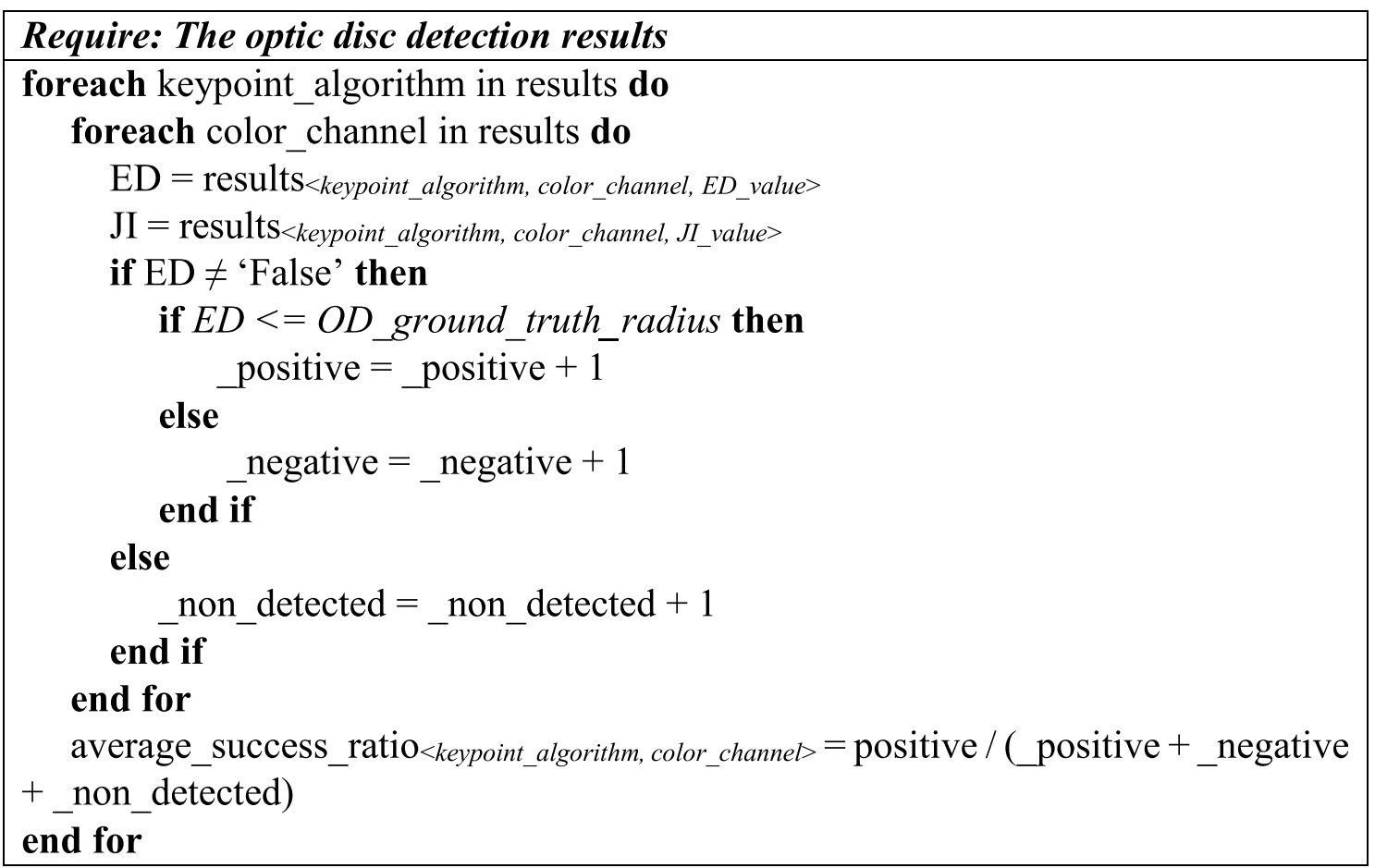

\section{Results}

Experiments were carried out on the publicly available DRIVE retinal fundus image dataset, which is widely used by many researchers. The retinal images consist of a variety of complex backgrounds, some occlusion, and variations in lighting. If the ED value is lower than the radius value, the detection of the OD region is accepted as successful. Table 1 presents the experimental results.

In this study, the effects of keypoint detection algorithms and color channels were evaluated. All image processing steps except the keypoint detection and color channel were the same for analysis of each retinal image. $92.5 \%, 92.5 \%$, and $92.5 \%$ success ratios were achieved using the keypoints detected by ORB, SIFT, and SURF algorithms, respectively on the RGB and green channel images. $47.5 \%, 37.5 \%$, and $42.5 \%$ success ratios were achieved using the keypoints detected by ORB, SIFT, and SURF algorithms, respectively on the red channel images. $32.5 \%, 30.0 \%$, and $30 \%$ success ratios were achieved using the keypoints detected by ORB, SIFT, and SURF algorithms, respectively on the blue channel images. According to the results, the proposed study was quite successful with the ORB, SIFT, and SURF keypoint detector algorithms on the RGB and green channel since 37 out of 40 optic disc regions detect successfully. So, we can say that noticeable results were obtained on the RGB and green channel.

Figure 6 presents the sample results within the frame of three categories that are successful-detected, falsedetected and non-detected. In Fig. 6a, two sample images
Table 1 Optic disc detection results on the DRIVE dataset

\begin{tabular}{|c|c|c|c|c|c|c|c|c|c|c|c|c|}
\hline & \multicolumn{4}{|c|}{ ORB } & \multicolumn{4}{|c|}{ SIFT } & \multicolumn{4}{|c|}{ SURF } \\
\hline & SD & FD & ND & $\%$ & SD & FD & ND & $\%$ & SD & FD & ND & $\%$ \\
\hline RGB & 37 & 2 & 1 & 92.5 & 37 & 2 & 1 & 92.5 & 37 & 2 & 1 & 92.5 \\
\hline Red & 19 & 4 & 17 & 47.5 & 15 & 3 & 22 & 37.5 & 17 & 3 & 20 & 42.5 \\
\hline Green & 37 & 2 & 1 & 92.5 & 37 & 2 & 1 & 92.5 & 37 & 2 & 1 & 92.5 \\
\hline Blue & 13 & 2 & 25 & 32.5 & 12 & 1 & 27 & 30.0 & 12 & 1 & 27 & 30.0 \\
\hline
\end{tabular}

$S D$ successful detected, $F D$ failed detected, ND non-detected, \% success rate, Bold font indicates the best results 
Fig. 6 Sample results

a Successful-detected
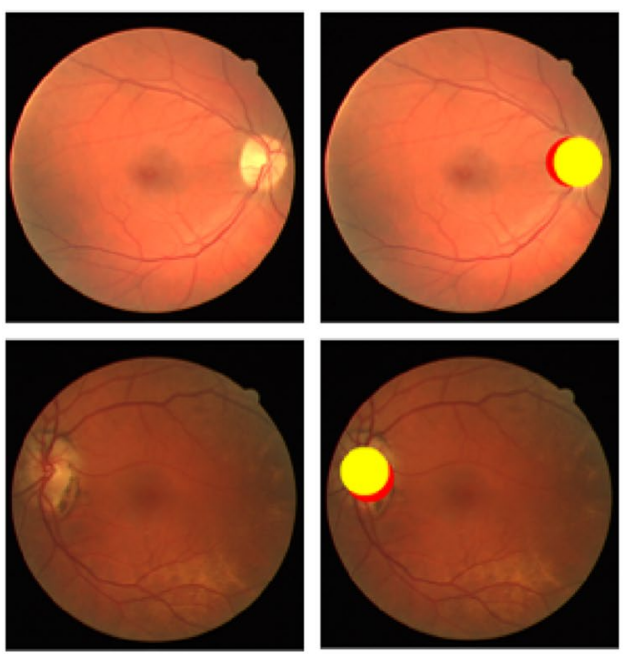

C Non-detected

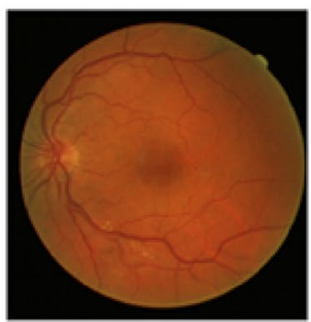

b False-detected
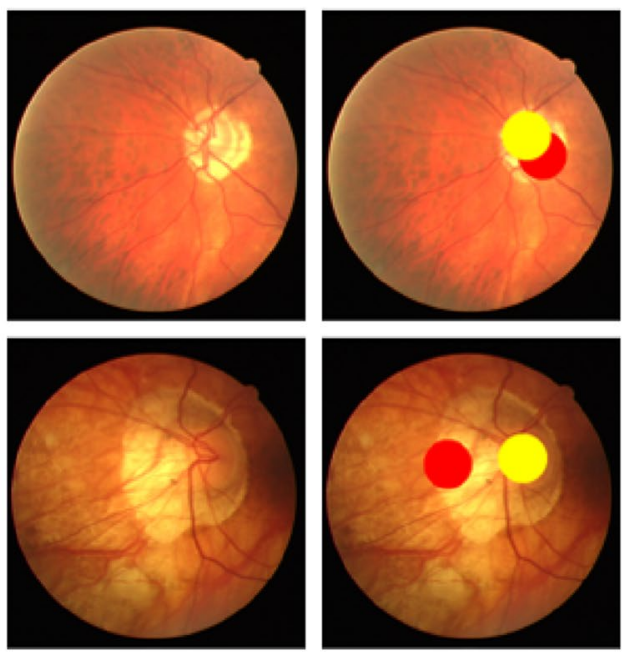

Note: Circle with yellow color: Groundtruth optic disc region

Circle with red color: Detected optic disc region

Table 2 Comparison with some of exiting methods in the literature on DRIVE dataset

\begin{tabular}{llc}
\hline Studies & Techniques & Success ratio (\%) \\
\hline Mitra et al. [2] & Convolution Neural Network & 99.41 \\
Muhammed [6] & Entropy and sliding window based techniques & 95.0 \\
Park et al. (2006) [9] & Threshold, detection of object roundness and circle detection & 90.25 \\
Zhu et al. [11] & Sobel operators and Hough transform & 90 (distance $<40$ pixels) \\
& & 95 (distance $<60$ pixels) \\
Abdullah et al. [13] & Circular Hough transform and grow-cut algorithm & 78.60 \\
Hamednejad and Pourghassem [14] & DBSCAN clustering algorithm & 78.18 \\
Roychowdhury [16] & Region-based features and supervised classification & 99.10 \\
Zahoor et al. [20] & Polar transform & 99.80 \\
Proposed approach & Image pre-processing, image processing, keypoint detection and & 92.50 (Best result) \\
\end{tabular}

The result of the proposed study is in bold

that were successfully detected in RGB and Green color spaces are given. In the two images in Fig. 6b, the optic disc region was detected incorrectly in the RGB and Green spaces as well as other color spaces. As can be seen in incorrectly detected images, it is thought that the distortions in the area of the optic disc caused this region to be detected incorrectly. Also, in these color spaces, no optic disc region was found in the image in Fig. $6 c$. The reason why the optic disc region cannot be detected in the image in Fig. $6 \mathrm{c}$ is thought to be due to the uncertainty of this region. Consequently, it can be concluded that image distortions and uncertainties make object detection difficult.

\section{Discussion}

For the sake of comparison, Table 2 summarizes the optic disc detection results achieved by our method and by other studies in the literature. As seen in this table, our proposed approach achieved an accuracy value of $92.5 \%$ 
on the publicly available DRIVE dataset and gave better performance than $[9,13,14]$. The studies of 9 and 13 are based on circle detection. The difference of our study from these studies is that it includes LBP texture analysis of a region around the identified keypoint. The study of 14 is a cluster-based study. But, the study of Convolution Neural Network [2], the study of entropy and sliding window techniques [6], the study of Sobel operators and Hough transform [11], the study of feature extraction and supervised classification [16] and the study of polar transform [20] presented slightly more successful than the texture analysis used in the proposed study. It can be stated that Convolution Neural Network, Entropy and sliding window, polar transform, and feature extraction and classification approaches are successful approaches to detect optic disc region. Although the performance of the proposed approach in this study is behind the studies of $[2,6,11,16$, $20]$, the proposed study offers a high success ratio of $92.5 \%$ and is acceptable for optic disc detection.

\section{Conclusion}

OD detection is an important prerequisite for the diagnosis of retinal diseases, i.e. diabetic retinopathy. In other words, accurate detection of OD is crucial in terms of image analysis and computer-aided diagnosis. The detection of the optic disc in the diseased retina such as diabetic retinopathy is more difficult than a healthy retina. Because the regions in the retinal image are candidate for being exudate in computer vision and machine learning systems. Therefore, the detection of this region must be carried out. The presented study introduced a texture analysis on the region of interest regarding detected keypoints on the different color spaces for detecting the best candidate optic disc region with validating performed using the ED and J on the DRIVE dataset. The experiments show that (a) keypoint detection is successful because one or more of them is on the optic disc region, (b) evaluation process based on local binary pattern method is accepted. The results demonstrate that the proposed study is effective for the automatic detection of the optic disc. The best average success ratio with a value of $92.5 \%$ was obtained on the RGB and green channel.

The limitation of this study is that the samples of patch images used in texture analysis are restricted. Providing domain adaptation by working with patch images from different sources for detecting the optic disc is among the goals. In addition, the performance of other keypoint algorithms on retinal images will be the subject of research. Another limitation of this study is that identifying the best candidate optic disc region for each key point requires repetitive execution of the same processes. It is a fact to be aware that if there are many key points, the time required to obtain the result will be increased.

In future work, by applying efficient image processing techniques and segmentation algorithms, the Jl value will be tried to be maximized and ED will be tried to be minimized. Finally, the studies of logarithmic image processing with low complexity are widely used in the literature [38-40]. In this context, the experiments including logarithmic image processing approaches having low complexity for increasing OD segmentation accuracy are among the targeted studies.

Acknowledgements The authors would like to thank the researchers who published the DRIVE retinal image dataset. A special thank goes to ophthalmologist Hasan Basri Çakmak for his support and assistance in optic disc ground-truth bounding box coordinates in each retinal image.

Funding Authors declare that this study no funded.

\section{Declarations}

Conflict of interest Authors declare no conflict of interest.

Open Access This article is licensed under a Creative Commons Attribution 4.0 International License, which permits use, sharing, adaptation, distribution and reproduction in any medium or format, as long as you give appropriate credit to the original author(s) and the source, provide a link to the Creative Commons licence, and indicate if changes were made. The images or other third party material in this article are included in the article's Creative Commons licence, unless indicated otherwise in a credit line to the material. If material is not included in the article's Creative Commons licence and your intended use is not permitted by statutory regulation or exceeds the permitted use, you will need to obtain permission directly from the copyright holder. To view a copy of this licence, visit http://creativecommons. org/licenses/by/4.0/.

\section{References}

1. Youssif AAHAR, Ghalwash AZ, Ghoneim AASAR (2008) Optic disc detection from normalized digital fundus images by means of a vessels' direction matched filter. IEEE Trans Med Imaging 27(1):11-18

2. Mitra A, Banerjee PS, Roya S, Roya S, Setuaa SK (2018) The region of interest localization for glaucoma analysis from retinal fundus image using deep learning. Comput Method Program Biomed 165:25-35

3. Fernandez-Granero MA, Sarmiento A, Sanchez-Morillo D, Jiménez S, Alemany P, Fondón I (2017) Automatic CDR estimation for early glaucoma diagnosis. J Healthcare Eng 2017:14

4. Zhang D, Yi Y, Shang X, Peng Y (2012) Optic disc localization by projection with vessel distribution and appearance characteristics. In: IEEE Proceedings of 2012 international conference on pattern recognition, Tsukuba, Japan, 11-15 November, pp 3176-3179

5. Bhuiyan A, Kawasaki R, Wong TY, Rao K (2009) A new and efficient method for automatic optic disc detection using geometrical features. In: World Congress on Medical Physics and 
Biomedical Engineering, Munich, Germany, 7-12 September, pp $1131-1134$

6. Muhammed L (2018) Localizing optic disc in retinal image automatically with entropy based algorithm. Int J Biomed Imaging 2018:7

7. Allam AMN, Youssif AAH, Ghalwash AZ (2015) Automatic segmentation of optic disc in eye fundus images: a survey. Electron Lett Comput Vis Image Anal 14(1):1-20

8. Akyol K, Şen B, Bayir Ş (2016) Automatic detection of optic disc in retinal image by using keypoint detection, texture analysis, and visual dictionary techniques. Comput Math Method Med 6814791:10

9. Rublee E, Rabaud V, Konolige K, Bradski G (2011) ORB: an efficient alternative to SIFT or SURF. In: IEEE International Conference on Computer Vision, ICCV, Barcelona, Spain, 6-13 November

10. Lowe DG (2004) Distinctive image features from scaleinvariant keypoints. Int J Comput Vis 60(2):91-110

11. Bay H, Ess A, Tuytelaars T, Gool LV (2008) SURF: Speeded-up robust features. Comput Vis Image Und 110:346-359

12. Lalonde M, Beaulieu M, Gagnon L (2001) Fast and robust optic disc detection using pyramidal decomposition and Hausdorff-based template matching. IEEE Trans Med Imaging 20(11):1193-1200

13. Park M, Jin JS, Luo S (2006) Locating the optic disk in retinal images. In: IEEE Proceedings of the International Conference on Computer Graphics, Imaging and Visualisation (CGIV '06), Sydney, Australia, pp 141-145

14. Mahfouz AE, Fahmy AS (2010) Fast localization of the optic disc using projection of image features. IEEE Trans Image Process 19(12):3285-3289

15. Zhu X, Rangayyan RM, Ells AL (2010) Detection of the optic nerve head in fundus images of the retina using the Hough transform for circles. J Digit Imaging 23:332-341

16. Whardana AK, Suciati N (2014) A simple method for optic disk segmentation from retinal fundus image. Int J Image Graph Signal Process 6(11):36-42

17. Abdullah M, Fraz MM, Barman SA (2016) Localization and segmentation of optic disc in retinal images using circular Hough transform and grow-cut algorithm. PeerJ 4(e2003):52

18. Hamednejad G, Pourghassem H (2016) Retinal optic disk segmentation and analysis in fundus images using DBSCAN clustering algorithm. In: IEEE 23rd Iranian Conference on Biomedical Engineering and 2016 1st International Iranian Conference on Biomedical Engineering (ICBME), Tehran, Iran, 24-25 November, pp 122-127

19. Ichim L, Popescu D (2016) Optic disc localization based on feature sorting. Memoirs Sci Sect Roman Acad Tome 36:63-70

20. Roychowdhury S, Koozekanani DD, Kuchinka SN, Parhi KK (2016) Optic disc boundary and vessel origin segmentation of fundus images. IEEE J Biomed Health Inform 20:1562-1574

21. Park JS, Cho HS, Cho J (2017) Automated extraction of optic disc regions from fundus images for preperimetric glaucoma diagnosis. In: IEEE 17th International Conference on Control, Automation and Systems (ICCAS), Jeju, Korea (South), 18-21 October, pp 1107-1110

22. Sharma A, Agrawal M, Lall B (2017) Optic disc detection using vessel characteristics and disc features. In: IEEE Twenty-third National Conference on Communications (NCC), Chennai, India, 2-4 March, pp 1-6

23. Sudhan GHH, Aravind RG, Gowri K, Rajinikanth V (2017) Optic disc segmentation based on Otsu's thresholding and level set.
In: IEEE International Conference on Computer Communication and Informatics (ICCCI), Coimbatore, India, 5-7 January, pp 1-5

24. Zahoor MN, Fraz MM (2017) Fast optic disc segmentation in retina using polar transform. IEEE Access 5:12293-12300

25. Al-Bander B, Williams BM, Al-Nuaimy W, Al-Taee MA, Pratt $\mathrm{H}$, Zheng $Y$ (2018) Dense fully convolutional segmentation of the optic disc and cup in colour fundus for glaucoma diagnosis. Symmetry 10(87):1-16

26. Fan Z, Rong Y, Cai X, Lu J, Li W, Lin H, Chen X (2018) Optic disk detection in fundus image based on structured learning. IEEE J Biomed Health Inform 22(1):224-234

27. Hou K, Liu N, Jia W, He Y, Lian J, Zheng Y (2018) Optic disc detection from fundus photography via best-buddies similarity. Appl Sci 8(709):1-12

28. Nergiz M, Akın M, Yıldız A, Takeş Ö (2018) Automated fuzzy optic disc detection algorithm using branching of vessels and color properties in fundus images. Biocybern Biomed Eng 38(4):850-867

29. Díaz-Pernil D, Fondón I, Peñ-Cantillana F, Gutiérrez-Naranjo MA (2016) Fully automatized parallel segmentation of the optic disc in retinal fundus images. Pattern Recogn Lett 83(1):99-107

30. Calimeri F, Marzullo A, Stamile C, Terracina G (2016) Optic disc detection using fine tuned convolutional neural networks. In: IEEE 12th International Conference on Signal-Image Technology \& Internet-Based Systems (SITIS), Naples, Italy, 28 November-1 December, pp 69-75

31. Yu S, Xiao D, Frost S, Kanagasingam Y (2019) Robust optic disc and cup segmentation with deep learning for glaucoma detection. Comput Med Imaging Graph 74:61-71

32. Wang L, Liu H, Lu Y, Chen H, Zhang J, Pu J (2019) A coarse-to-fine deep learning framework for optic disc segmentation in fundus images. Biomed Signal Process Control 51:82-89

33. DRIVE (2018) Available at: https://www.isi.uu.nl/Research/Datab ases/DRIVE/

34. Pisano ED, Zong $S$, Hemminger BM, DeLuca M, Johnston RE, Muller K, Braeuning MP, Pizer SM (1998) Contrast limited adaptive histogram equalization image processing to improve the detection of simulated spiculations in dense mammograms. J Digit Imaging 11(4):193-200

35. Vimal SP, Thiruvikraman PK (2012) Automated image enhancement using power law transformations. Sadhana 37(6):739-745

36. Ojala T, Pietikäinen M, Harwood D (1996) A comparative study of texture measures with classification based on feature distributions. Pattern Recognit 29(1):51-59

37. Rahman M, Hassan MR, Buyya R (2010) Jaccard Index based availability prediction in enterprise grids. Procedia Comput Sci 1(1):2707-2716

38. Jourlin M, Pinoli JC (2001) Logarithmic image processing: the mathematical and physical framework for the representation and processing of transmitted images. Adv Imaging Electron Phys 115:129-196

39. Florea C, Albu F, Vertan C, Drimbarean A (2008) Logarithmic tools for in-camera image processing. In: IET Irish Signals and Systems Conference (ISSC 2008), Galway, 18-19 June, pp 394-399

40. Albu F, Vertan C, Florea C, Drimbarean A (2009) One scan shadow compensation and visual enhancement of color images. In: 16th IEEE International Conference on Image Processing (ICIP), Cairo, Egypt, 7-10 November, pp 3133-3136

Publisher's Note Springer Nature remains neutral with regard to jurisdictional claims in published maps and institutional affiliations. 